\title{
A VLSI Model of the Bat Dorsal Nucleus of the Lateral Lemniscus for Azimuthal Echolocation
}

\author{
Rock Z. Shi \\ Electrical and Computer Engineering Department \\ Institute for Systems Research \\ University of Maryland \\ College Park, MD 20742,USA \\ Email: rshi@glue.umd.edu
}

\author{
Timothy K. Horiuchi \\ Electrical and Computer Engineering Department \\ Institute for Systems Research \\ Neuroscience and Cognitive Science Program \\ University of Maryland \\ College Park, MD 20742,USA \\ Email: timmer@isr.umd.edu
}

\begin{abstract}
The dorsal nucleus of the lateral lemniscus (DNLL) is a distinct group of auditory cells that play a strategic role in azimuthal echolocation in the bat. Dominated by EI-type cells that receive excitation from the contralateral ear and inhibition from the ipsilateral ear, the DNLL processes interaural level difference (ILD) information by integrating inputs from lower brainstem areas and projecting its outputs to the midbrain. In this paper, we propose a two layer recurrent spiking neural network model that simulates ILD processing by the DNLL, and present a VLSI implementation using the address-event representation (AER) protocol. We demonstrate, using this neuromorphic VLSI-based hardware system, that long-lasting inhibition in the DNLL can alter its spatial selectivity to multiple sounds (objects).
\end{abstract}

\section{INTRODUCTION}

Bats perceive the world by emitting ultrasonic pulses and localizing the resulting echoes from objects. This sensory modality is called echolocation. Their small head size and the use of high frequency sound make the interaural level differences (ILDs) their primary cue for azimuthal echolocation. ILDs are known to be coded at the earliest stage of binaural processing by neurons in the lateral superior olive (LSO). The LSO receives its principal excitatory (E) inputs from the ipsilateral ear (from the same side as the LSO) and inhibitory (I) inputs from the contralateral (opposite side) ear. The projections from each LSO are bilateral and terminate in both the ipsilateral and contralateral DNLL. The ipsilateral projections from the LSO are mainly inhibitory. In contrast, the crossed projections from the LSO to DNLL are excitatory. Like the LSO, the DNLL mainly contains EI type neurons (cells that receive excitation from one ear and inhibition from the other ear). Fig. 1 shows the ILD pathway up to DNLL.

The DNLL is distinguished from the LSO by at least two features. First, the DNLL is dominated by GABAergic neurons, such that projections from the DNLL are mainly inhibitory. Second, unlike LSO, the DNLL on each side of the midline projects inhibition reciprocally (via the commissure of Probst). Stimulation of the inhibitory ear can evoke inhibition on a DNLL cell that can persist as long as $50 \mathrm{msec}$ after the stimulus has ended [1]. This long lasting inhibition indicates that the DNLL must play an important role in the ILD processing of multiple sounds. In fact, due to the connections from LSO to DNLL, which are described as "push-pull" [1],
[2], an initial binaural sound that favors the ipsilateral ear should suppress the responses to trailing sounds that normally would be excitatory if they were presented alone.

In spite of the importance of the DNLL in auditory processing, there are only a few detailed biological models that include the DNLL, owing perhaps to the lack of neurophysiological data from the DNLL. In one example, Reed and Blum studied steady-state DNLL responses to binaural stimuli based on a firing rate model [3], [4]. In this paper, we propose a twolayer recurrent spiking neural network model that enables us to simulate ILD processing by the DNLL, and an AER-based chip implementation of such a model. We demonstrate, with this neuromorphic bat ILD processing system, how the DNLL responds differentially to spatially-separated sound sources depending upon their order of arrival. This paper continues initial work by Horiuchi and Hynna on a spike-based VLSI modeling study of the bat ILD system [5], and more recent work by Shi and Horiuchi on a VLSI LSO model [6].

\section{Model, Circuit AND System}

\section{A. Network model}

We propose a two-layer spiking neural network model for ILD processing in the bat LSO and DNLL. Our model is tuned

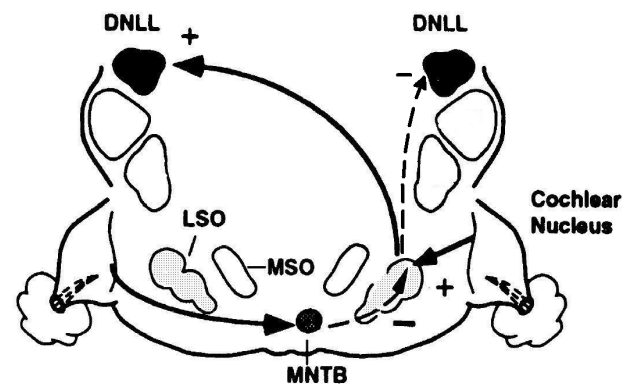

Fig. 1. ILD pathway to DNLL. The ILD pathway starts from cochlear nucleus (CN). The first ILD processing center is the LSO, which receives excitation from the ipsilateral $\mathrm{CN}$ but inhibition from the contralateral $\mathrm{CN}$ via the medial nucleus of the trapezoid body (MNTB). The second ILD processing center is the DNLL, which receives inhibition from the ipsilateral LSO (as well as from cochlear nucleus $(\mathrm{CN})$ ), but the excitation from the contralateral LSO $(\mathrm{CN})$. Solid line for excitation, dotted line for inhibition. Revised from [1]. 


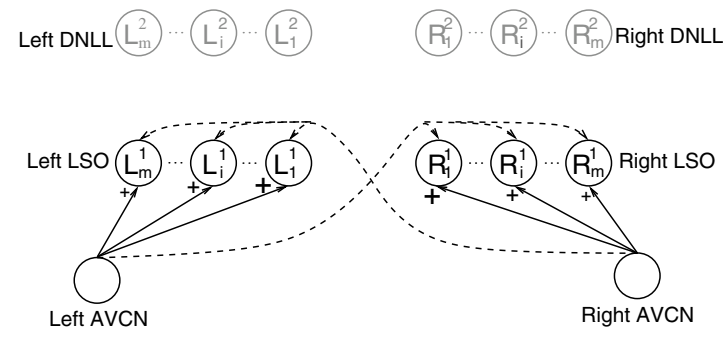

(a) The LSO population array.

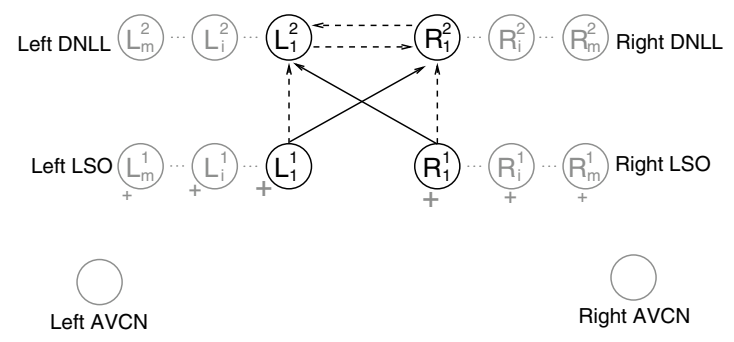

(b) The two layer connection.

Fig. 2. The LSO-DNLL two layer network model. (a) The LSO population array. The LSO layer consists of two m-neuron arrays, one for the left, one for the right. To generate population response, the excitatory synaptic strength of the LSO array are set such that it decreases as the cell number increase. The LSO receives input from the AVCN, the output from the front end. (b) The LSO-DNLL interconnection. A DNLL cell, e.g $\mathrm{L}_{\mathrm{i}}^{2}(\mathrm{i}=1, \ldots, \mathrm{m})$, receives excitation from the contralateral $\operatorname{LSO}\left(\mathrm{R}_{\mathrm{i}}^{1}\right)$, inhibition from the ipsilateral LSO $\left(\mathrm{L}_{\mathrm{i}}^{1}\right)$. It also inhibits the opposite DNLL, $\mathrm{R}_{\mathrm{i}}^{2}$. Solid line for excitation, dotted line for inhibition.

to VLSI implementation, and emphasizes the processing and transformation of spike timing. Fig. 2(a) shows the sensory inputs to the LSO layer which come from the anterior ventral cochlear nucleus (AVCN). Each LSO cell consists of one spiking neuron with two synapses: one excitatory synapse for the input from the ipsilateral AVCN, and one inhibitory synapse for the input from the contralateral AVCN. Fig. 2(b) describes the LSO-DNLL two-layer structure. Each DNLL cell consists of one spiking neuron driven by 3 synapses: one excitatory synapse receiving excitatory input from the contralateral LSO, one inhibitory synapse receiving inhibitory input from the ipsilateral LSO, and one inhibitory synapse receiving input from the opposite DNLL.

\section{B. Circuit implementation}

We chose a compact and easily controllable synapse circuit that was first introduced by Lazzaro and Wawrzynek [7]. The synapse circuits are shown in Fig. 3. When $V_{w}$ is set below the transistor threshold voltage, this synapse produces a clean exponential decay of the synaptic current, which is an important aspect of the biological counterpart.

The neuron circuit schematic is shown in Fig. 4. This circuit implements an integrate-and-fire neuron model with constant leakage, and a controllable refractory period. The spikes are transmitted from the chip using the address-event

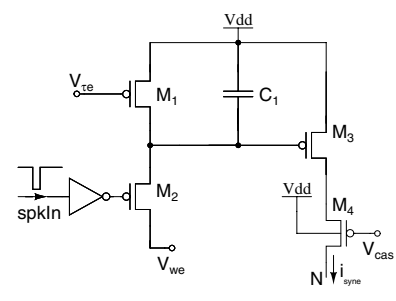

(a)

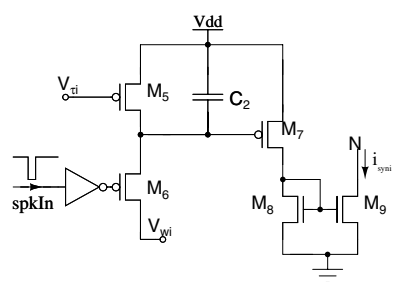

(b)
Fig. 3. Synapse circuit. (a) Excitatory synapse circuit. (b) Inhibitory synapse circuit. $V_{\tau e}$ and $V_{\tau i}$ set the excitatory and inhibitory synaptic time constants, respectively. $V_{w e}$ and $V_{w i}$ set the excitatory and inhibitory synaptic strength, respectively. $\mathrm{M}_{4}$ and $V_{\text {cas }}$ are for isolating neuron's membrane voltage.

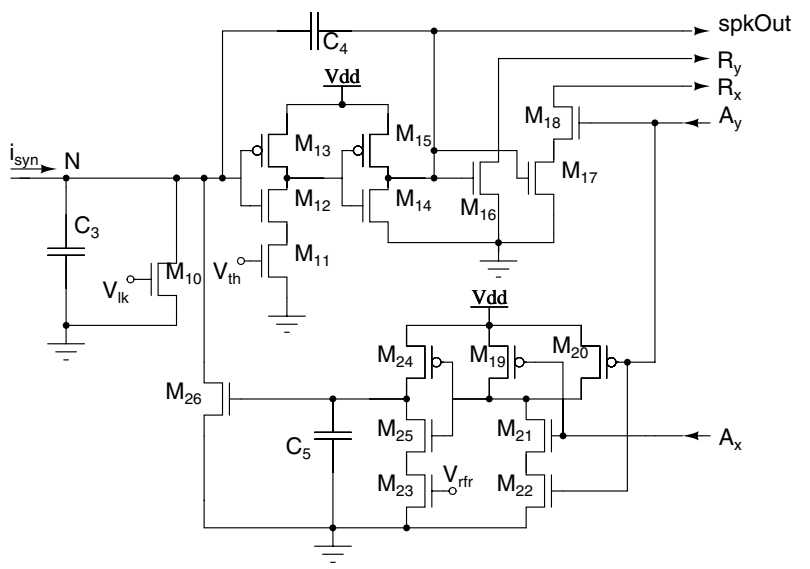

Fig. 4. Neuron circuit. The neuron integrate synaptic current through membrane capacitance $\mathrm{C}_{3}$, generate spikes through $\mathrm{M}_{11}-\mathrm{M}_{15}$ and capacitor $\mathrm{C}_{4}$ , and generates AER $X$ and $Y$ request signals $R_{x}$ and $R_{y}$ by $M_{16}-M_{18}$. The neuron's refractory period is set by $\mathrm{M}_{20}-\mathrm{M}_{26}$ with capacitor $\mathrm{C}_{5} . V_{t h}$ sets the neuron's spiking threshold, $V_{l k}$ sets membrane leakage, $V_{r f r}$ sets the neuron's refractory period.

representation protocol, which overcomes the limited number of available pads and allows modularization for multi-chip designs. The AER system we used is similar to that described in [8], but without the pipelining. We omit details of the AER circuitry in this paper. The neuron circuit can be divided into four functional parts. The first part consists of the membrane capacitance $\mathrm{C}_{3}$ and a constant current leak by transistor $\mathrm{M}_{10}$. The second part, including transistors $\mathrm{M}_{11}-\mathrm{M}_{15}$ and capacitor $\mathrm{C}_{4}$, is the spike generator. The spiking threshold is determined by $V_{t h}$ and $V d d$. spkOut is the spike output. The third part, including $\mathrm{M}_{20}-\mathrm{M}_{26}$ with capacitor $\mathrm{C}_{5}$ sets the neuron's refractory period. The fourth part, $\mathrm{M}_{16}-\mathrm{M}_{18}$ generates the $\mathrm{X}$ and $\mathrm{Y}$ request signals for the 2-D AER system.

\section{Chip and neuromorphic System}

The circuit was fabricated in a commercially-available 1.5 $\mu m$ technology. The chip contains 32 LSO neurons (16 for the left LSO and 16 for the right LSO) and 32 DNLL neurons (16 for the left DNLL and 16 for the right DNLL).

The AVCN outputs are considered the sensory "front-end" for this chip, generating bursts of spikes whose rate encodes 
the sonar-generated echo intensity at any given moment. The sonar transmits short $40 \mathrm{kHz}$ acoustic pulses (about $1 \mathrm{msec}$ ) using a speaker and then receives the returning echo signals. The received echo signals are amplified, half-wave rectified and then the envelope is extracted. The last stage of processing is a high-rate spike generator that represents the response of the population of neurons in the AVCN. We omit details of the front-end sonar components in this paper. This echolocation front-end has been described previously in [5], [6].

\section{TESTING Results}

\section{A. LSO response to one object and to two objects}

We first show a typical LSO population response to one object and then to two objects. In this experiment, we chose two vertical cylinders with the same diameter of $4.5 \mathrm{~cm}$. We performed five trials for each condition, using a large intertrial interval to avoid any interactions between trials. We first put object \#1 20 $0^{\circ}$ left of center, $55 \mathrm{~cm}$ away. The top panel of Fig. 5 shows the population response. Since the object is located to the left, all of the left LSO cells fire in all five trials, with cell 1 firing first and cell 16 last. Seven of the right LSO cells fire as well. The middle panel shows the response when object $\# 2$ is located $20^{\circ}$ right of center, $80 \mathrm{~cm}$ away. In this case, twelve cells fire, with cell 12 firing only two times, cell 11 firing three times, and cells 1-10 firing five times in five trials.
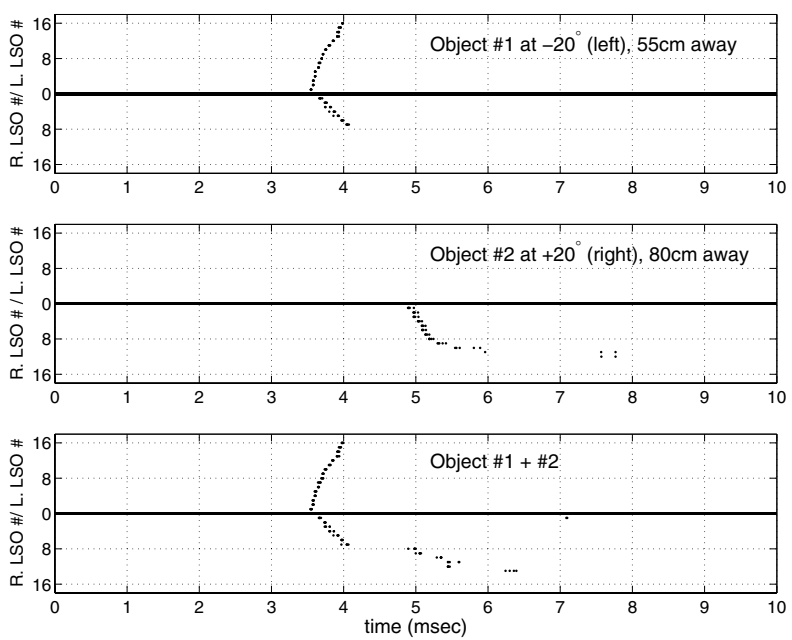

Fig. 5. LSO population response to single and multiple (2) objects. Top: Object \#1 alone is located at $20^{\circ}$ left to and $55 \mathrm{~cm}$ away from the speaker. Middle: Object \#2 alone is located at $20^{\circ}$ right to and $80 \mathrm{~cm}$ away from the speaker. Bottom: Object \#1 and object \#2 are both present. Both objects are a vertical cylinder with diameter about $4.5 \mathrm{~cm}$. Shown are raster plots with 5 trials

We then presented the two objects simultaneously. We observe that because object \#2 is further away than object $\# 1$, it will not change the system's response to the first object. Object \#1, however, does affect the right LSO's response to object \#2 in at least two significant ways. First, cells 1-7, which fired when object \#2 was presented alone, no longer fire. We point out that these missing spikes are a result of the refractory period in the neurons. As cells 1-7 of the right LSO fire due to the closer object (\#1), they are unable to respond to the second object, whose echo occurs within their refractory period. Second, cell 13 of the right LSO, which did not fire when object \#2 was presented alone, is now activated. This is due to residual (but decaying) membrane charge leftover from the inputs during the first object.

\section{B. The push-pull effect on DNLL}

Although there are 32 fully-connected DNLL cells, we do not report the population response of the DNLL in this paper, rather, we present a single DNLL cell response to study the distinguishing feature of the DNLL, the so-called "push-pull" effect that results from the mutual inhibition of the two DNLLs across the midline [1], [2]. For example, when an ipsilateral sound (a sound that is from the same side as the DNLL) occurs before a contralateral sound, the cross-inhibition will suppress the later sound that would normally be excitatory if it was presented alone.

In our experiment design, a preceding sound was obtained by putting an object $+35^{\circ}$ right of center and $50 \mathrm{~cm}$ away, while the trailing sound was created by an object at $-30^{\circ}$ of center and $70 \mathrm{~cm}$ from the speaker.

Fig. 6 shows the response to the further object (trailing sound) alone. The top panel shows the echo envelope signal from the left microphone. The middle panel shows the envelope signal for the right microphone. The object is located at left $30^{\circ}$ so the intensity on the left is stronger, driving the left LSO cell to fire at $4.97 \mathrm{msec}$ (the first marker dot in Fig. 6 above each trace). The left LSO cell projects excitation across the midline to the right DNLL cell which integrates the excitatory current (bottom trace) and fires at $5.71 \mathrm{msec}$ (the second marker dot above each trace).
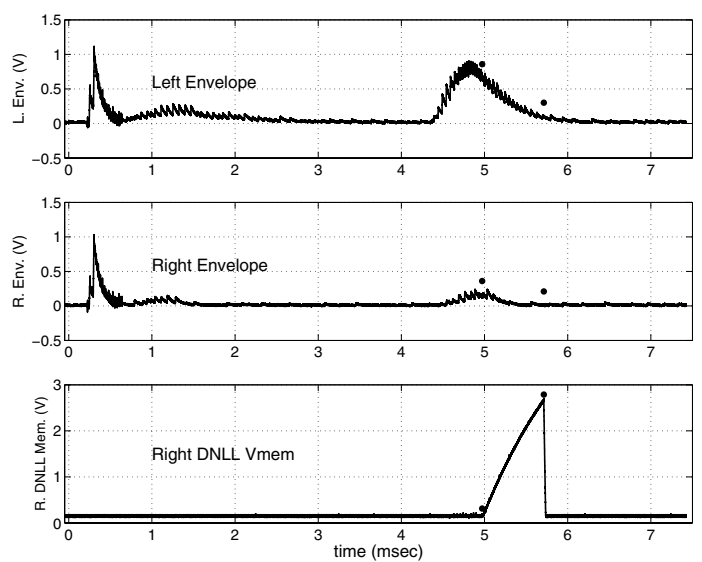

Fig. 6. Sample traces for response to trailing sound alone. Top: The left envelope signal. Middle: The right envelope signal. Bottom: The membrane voltage of the right DNLL. In all the three panels, the two marker points label the spiking time. The first point marks the time when the left LSO fires, and the second point marks the time the right DNLL cell fires.

Fig. 7 shows the response to the further object (trailing sound) when a closer object (preceding sound) was present. The close object, located $+35^{\circ}$ right of center drives the right LSO cell to fire at $3.96 \mathrm{msec}$ (the first marker dot above each trace). This right LSO cell projects excitation across the 

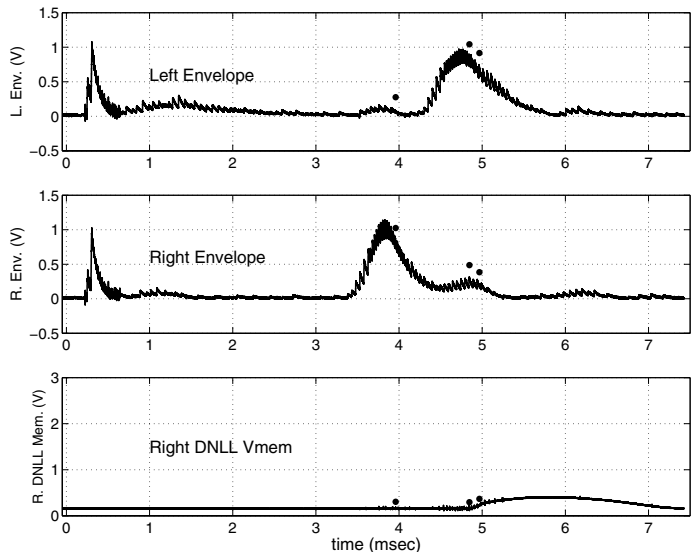

Fig. 7. Sample traces for response to both preceding and trailing sounds present. Top: The left envelope signal. Middle: The right envelope signal. Bottom: The membrane voltage of the right DNLL. In all the three panels, the three marker points label the spiking time. The first point marks the time when the right LSO fires, the second point marks the time the left DNLL cell fires, and the third marks the time that the left LSO fires.

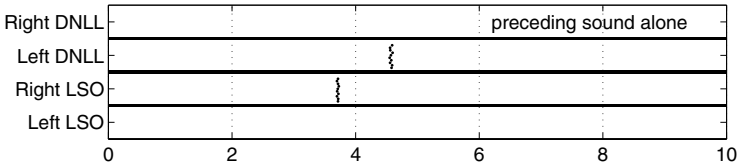

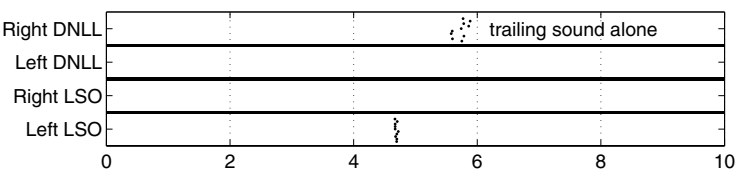

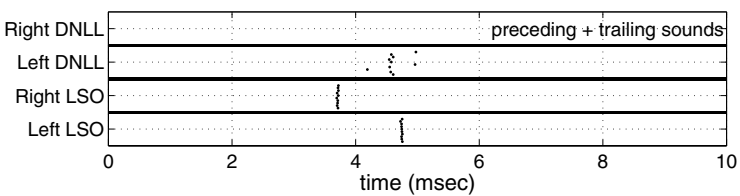

Fig. 8. DNLL push-pull response raster plots for 10 trials. Top: Preceding sound alone. As the preceding sound object is located at right $\left(+35^{\circ}\right)$, the right LSO cell fires and in turn excites the left DNLL. Middle: Trailing sound alone. As the trailing sound object is located at left $\left(-30^{\circ}\right)$, it excites the the left LSO cell and in turn to drive the right DNLL cell to fire. Bottom: When both sounds are present, the right DNLL cell is inhibited from the opposite DNLL cell (left DNLL).

midline to the left DNLL cell and causes the left DNLL cell to fire at $4.85 \mathrm{msec}$ (the second marker dot above each trace). The left DNLL cell sends the inhibition to the opposite DNLL (the right DNLL), so that the right DNLL cannot fire in response to excitation from the left LSO cell (the left LSO cell fires at $4.96 \mathrm{msec}$, the third marker dot).

Fig. 8 shows the raster plots for ten trials of the above experiment. In this plot it is easy to see the temporal relationship between the LSO and DNLL. In the multiple target case (bottom), the right DNLL cell, whose excitation comes from the left LSO, has been totally inhibited by the opposite DNLL. The time duration of the inhibition is clearly a function of the time constant of the inhibitory synapse and the recovery time of the neuron.

This push-pull connections in the DNLL play an important role in processing multiple sounds. In this particular experiment, what we have shown is that a preceding sound may have significant effects on the DNLL's response. This feature of the DNLL will affect the spatial selectivity of the inferior colliculus, the next stage of the ILD pathway, for multiple objects [1], [2].

\section{Conclusions}

In this paper we have demonstrated a functioning hardware model of portions of the bat LSO and DNLL network in response to real-life sonar echoes and spiking representations. We demonstrate that the response of these anatomicallyjustified network models to realistic stimuli is not a simple linear addition of the responses to single stimuli. In one case, we show the effects of a simple refractory period and in another case, we show the effect of long-lasting inhibition at the level of the DNLL. In prior work, we showed similar effects of persistent inhibition on the LSO. The time constants of synaptic currents and membrane potentials are larger than a millisecond, whereas usable echo information about independent objects in space arrives at the bat with separations on the order of a millisecond.

The ILD-selective responses of the LSO and DNLL are viewed to be the basis of azimuthal localization in many mammals at high frequencies and these results suggest the need for a more sophisticated view of how animals like bats might process ILD information from moving or from multiple objects.

\section{ACKNOWLEDGMENT}

This work is supported by the Air Force Office of Scientific Research (AFOSR-FA95500410130) and by the National Science Foundation (CCF0347573). We thank MOSIS for fabrication services in support of our neuromorphic analog VLSI course and teaching laboratory.

\section{REFERENCES}

[1] L. Yang and G. D. Pollak, "The roles of GABAergic and glycinergic inhibition on binaural processing in the dorsal nucleus of the lateral lemniscus of the mustache bat," J. Neurophysiol., vol. 71, pp. 1999-2013, 1994.

[2] — - "Features of ipsilaterally evoked inhibition in the dorsal nucleus of the lateral lemniscus," Hear. Res, vol. 122, pp. 125-141, 1998.

[3] M. C. Reed and J. J. Blum, "Model calculations of steady-state responses to binaural stimuli in the dorsal nucleus of the lateral lemniscus," Hear. Res., vol. 136, pp. 13-28, 1999.

[4] J. J. Blum and M. C. Reed, "Model calculations of time dependent responses to binaural stimuli in the dorsal nucleus of the lateral lemniscus," Hear. Res., vol. 149, no. 1-2, pp. 77-90, 2000.

[5] T. Horiuchi and K. Hynna, "Spike-based VLSI modeling of the ILD system in the echolocating bat," Neural Networks, vol. 14, pp. 755-762, 2001.

[6] R. Z. Shi and T. K. Horiuchi, "A VLSI model of the bat lateral superior olive for azimuthal echolocation," in Proc. ISCAS'04, vol. 4, May 2004, pp. 900-903.

[7] J. P. Lazzaro, "Low-power silicon axons, neuons, and synapses," in Silicon Implementations of Pulse Coded Neural Networks, M. E. Zaghloul, J. L. Meador, and R. W. Newcomb, Eds. Norwell, MA: Kluwer Academic Publishers, 1994, pp. 153-164.

[8] K. A. Boahen, "Point-to-point connectivity between neuromorphic chips using address-events," IEEE Trans. Circuits Syst. II, vol. 47, no. 5, pp. 416-434, 2000. 